Ann. Abeille, I964, $7(4), 365-372$

\title{
L'UTILISATION DES CARTES PERFORÉES POUR L'ANALYSE POLLINIQUE DES MIELS
}

\author{
J. LOLVE.LX \\ Station de Recherches sur l'Abeille et les Insectes sociaux. \\ Bures-sur-Yvette (Seine-et-Uise)
}

SOMMAIRE

L'auteur expose les possibilités offertes par un système classique de cartes perforées (Sphinxo) pour le classement des données fournies par l'analyse pollinique des miels. Ces cartes permettent de retrouver très rapidement tous les miels présentant un spectre pollinique déterminé. Elles sont également utilisables pour plusieurs autres types de recherches.

La manipulation des données fournies par l'analyse pollinique pose des problèmes de plus en plus compliqués. Fn effet, au fur et à mesure que les miels des différentes régions du globe font l'objet d'études particulières, les informations que l'on est susceptible d'utiliser pour la détermination de l'origine géographique d'un miel sont de plus en plus nombreuses et complexes. Il est donc normal qu'on ait songé à se servir des cartes perforées pour faciliter le travail d'interprétation des spectres polliniques.

I,e système que nous avons étudié est celui des cartes perforables sur toute leur surface dites "Sphinxo ». Si nous disposons, par exemple, de 2500 miels (les cartes peuvent recevoir 5 ooo perforations) nous les enregistrons tout d'abord dans un cahier, ce qui permet de leur attribuer un numéro d'ordre. L'ordre est généralement chronologique puisque les miels sont enregistrés à leur arrivée au laboratoire. A chaque numéro d'ordre correspond sur la carte un emplacement déterminé où il est possible de faire une perforation ronde de $\mathrm{I}, 5 \mathrm{~mm}$ de diamètre au moyen d'un poinçon. On dispose d'une table de perforation permettant de fixer rigoureusement les coordonnées du trou. Les perforations se font en fonction d'une caractéristique déterminée et les cartes sont établies à raison d'une par caractéristique.

Si nous prenons commé caractéristique le lieu d'origine des échantillons, nous pourrons établir une carte par partie du monde ou par pays ou par département ou 
par commune, selon la nature du travail prévu. La carte "France " se trouvera perforée à l'endroit de tous les numéros correspondant à des miels produits en France ; la carte Mexique, la carte Espagne, etc. porteront de la même façon les perforations correspondant aux numéros des miels mexicains, espagnols, etc.

Comme il est possible de perforer jusqu'à 6 cartes à la fois on peut très bien perforer d'un seul coup plusieurs cartes correspondant à des aires géographiques de plus en plus vastes. Premier exemple : soit à porter sur carte le miel $\mathbf{n}^{0}$ I 98 originaire du nord-ouest du département de la Seine-et-Marne; on pourra perforer d'un seul coup :

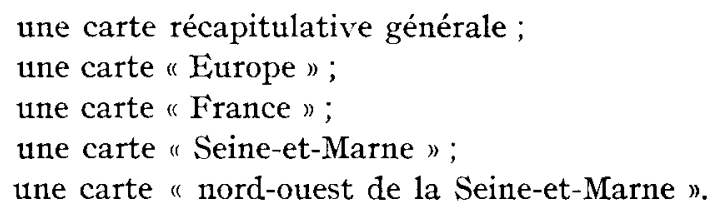

Deuxième exemple : soit à porter sur carte le miel $\mathrm{n}^{0} 624$ originaire du Mexique ; on pourra perforer d'un même coup :

la carte récapitulative générale déjà citée à l'exemple $\mathrm{n}^{0} \mathrm{I}$;

la carte "Le monde moins la France ";

une carte " Amériques ";

une carte "Mexique".

Lorsque nos 2500 échantillons de miels auront été mis sur cartes perforées, nous disposerons de quelques dizaines de cartes, Ioo à 150 au maximum, dont chacune constituera une liste complète de tous les échantillons ayant une origine commune, origine très large (Amériques) ou étroite (Seine-et-Marne) ou très étroite si nous le jugeons utile (nord-ouest de la Seine-et-Marne). La consultation de ces fiches permet de retrouver instantanément tous les miels d'une origine déterminée. Si nous recherchons seulement les miels du nord du Mexique et que nous n'ayons pas fait de fiche pour cette région notre recherche sera cependant simplifiée puisqu'il suffira de trier parmi les miels mexicains figurant sur la carte "Mexique".

En dehors de l'origine géographique, il est possible d'établir des cartes perforées pour n'importe quelle caractéristique. Il suffit de posséder les données que l'on désire utiliser, soit pour la totalité, soit même pour une partie seulement des échantillons. C'est ainsi qu'il est possible d'établir des cartes pour des données telles que :

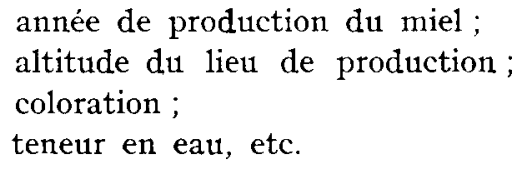

En ce qui concerne le spectre pollinique des miels, il est relativement aisé de le mettre sur cartes, soit partiellement, soit en totalité. Il suffit pour cela d'établir des cartes par plante (espèce, genre ou famille selon les cas) et de perforer les numéros des échantillons qui possèdent la plante en question dans leur spectre. $L_{T} a$ carte "Saliia " par exemple nous permettra de retrouver instantanément tous les miels qui contiennent le pollen de sauge dans leur spectre pollinique.

L'intérêt principal des cartes perforées ne réside toutefois pas dans l'établisse- 
ment de catalogues, si utiles soient-ils. En effet, la superposition de deux cartes représentant deux caractéristiques différentes met en évidence de façon immédiate les corrélations qui peuvènt exister entre ces caractéristiques. Si nous superposons la carte "Seine-et-Marne " et la carte "Sainfoin ", la lumière de la tablé de lecture lumineuse passera partout où les perforations concordent, c'est-à-dire que nous lirons directement les numéros de tous les miels de Sainfoin de Seine-et-Marne. Nous arons pris là un exemple très simplè ; en fait, il est possible d'aller beaucoup plus loin puisqu'on peut superposer au moins 6 à I 2 cartes, ce qui permet de composer tout un élément de spectre et de trouver quels sont les miels qui le possèdent, par simple lecture.

I)e même qu'il est possible de perforer d'un seul coup des cartes correspondant à des aires géographiques de plus en plus restreintes on peut perforer des séries de cartes " par plante " en faisant intervenir des éléments de classification de plus en plus étroits. Ainsi, par exemple, une plante telle que Contaurea jacea pourra figurer sur la série de cartes:

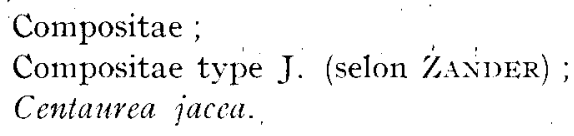

Signalons enfin qu'il est parfaitement possible d'établir pour chaque plante importante une série de cartes en fonction de la classe de fréquence. Nous aurons ainsi la série :
Sainfoin dominant.
Sainfoin accompagnement.
Sainfoin isolé.
Sainfoin récapitulation.

Combinée avec l'une quelconque des trois premières cartes, la carte récapitulative permet d'explorer deux classes ou groupes de classes à' la föis si nous superposons les cartes:

\author{
Sainfoin récapitulation. \\ France. \\ Sainfoin isolé:
}

Tous les miels français contenant du pollen de sainfoin à l'état isolé apparaittront de façon directe par superposition des 3 perforations mais tous les miels français à Sainfoin dominant ou d'accompagnement seront également mis en évidence sur le fond semi-transparent de la carte "Sainfoin isolé " qui fait écran.

Tel que nous venons de le décrire et compte tenu de ses multiples possibilités, le système Sphinxo appliqué à l'analyse pollinique des miels permet:

Io I'établir des catalogues permanents, faciles à compléter ou à rectifier et susceptibles de correspondre à toutes les caractéristiques possibles, allant du lieu d'origine à la composition chimique en passant par le spectre pollinique.

$2^{0}$ De rechercher rapidement des corrélations entre deux ou plusieurs caractéristiques et de chiffrer le taux de corrélation.

$3^{0}$ De rechercher tous les miels présentant une combinaison donnée de caractéristiques. Iin fait, il devient possible, connaissant un spectre pollinique de retronver 
en quelques instants tous les miels possédant la même combinaison, y compris ceux dont on aura relevé les caractéristiques dans la bibliographie. En effet, rien ne s'oppose à la mise sur cartes de tous les éléments épars dans les différentes publications.

Le travail de préparation des cartes peut paraitre considérable. Èn fait, il est rapide lorsque les données à inscrire sous forme de perforations sont bien préparées. C'est donc la préparation des données qui est longue, mais cette préparation est nécessaire quelque soit le système de cartes adopté, $\mathrm{y}$ compris les cartes pour machines électroniques I.B.M. ou Bull.

Notons encore qu'un travail incomplet reste utilisable. L'essentiel est de hiérarchiser. Si l'on possède 25 ou 30 miels des U. S. A. par exemple, il est totalement inutile de prévoir des cartes par État. Une carte globale "U. S. A. " est suffisante. De mème, en ce qui concerne les plantes à porter sur cartes : elles n'ont pas toutes la même importance et beaucoup peuvent ne faire l'objet que d'une seule inscription; c'est le cas, par exemple de Polygonum bistorta qui n'intervient pratiquement que comme pollen isolé et qu'on ne trouve que dans des miels bien particuliers. Par ailleurs, il est toujours possible d'opérer des regroupements de plantes sur un genre ou sur une famille. En opérant de cette façon, le nombre final des cartes reste dans les limites acceptables.

Il faut encore signaler que le système de cartes perforées que nous préconisons peut s'adapter à bien d'autres types de recherches. Voici quelques exemples :

Io Recherche du lieu de production théoriquement possible d'un miel dont on connâ̂t le spectre ou d'un type de miel déterminé. Les cartes sont établies par plante et on prépare par ailleurs une liste de lieux géographiques. Cette liste peut parfaitement correspondre à un quadrillage basé sur les méridiens et les parallèles. Pour chaque plante, on perfore tous les lieux où elle est présente. Pour simplifier le travail, on peut facilement changer l'échelle selon les parties du monde et représenter l'Asie par 5 ou ro numéros alors qu'un pays bien étudié demandera 50 ou 100 numéros différents. En superposant les cartes correspondant aux plantes d'un spectre énigmatique, on découvre instantanément quels sont les lieux du monde ò̀ ce spectre est possible, ce qui restreint le secteur des investigations. La plus grosse difficulté réside dans la nécessité de connaître avec précision la répartition géographique des espèces intéressantes; pour beaucoup de plantes, il s'agit là d'un travail bibliographique important.

$\mathbf{2}^{\circ}$ Détermination d'un pollen inconnu. Il ne peut s'agir dans ce cas que d'un travail d'approche mais qui serait très utile à chaque fois qu'on se trouve en présence d'un pollen énigmatique. Le premier travail consiste à dresser une liste de tous les pollens que l'on considère comme utiles aux recherches. Les cartes sont ensuite perforées en fonction des caractères morphologiques : forme, dimensions, apertures, exine, etc. En superposant les cartes correspondant à chaque caractéristique retenue, on peut restreindre considérablement le champ des recherches: la lumière n'apparait que pour un nombre limité de pollens parmi lesquels il convient de poursuivre le travail d'investigation.

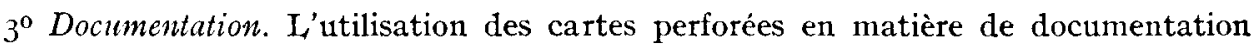
est suffisamment connue pour que nous n'insistions pas sur cet aspect du problème. Dans le système Sphinxo, les cartes sont établies par sujet et on numérote les documents. 
Il serait encore possible de développer le sujet et d'apporter d'autres précisions sur certaines modalités d'utilisation des cartes perforées. Je pense plus utile de montrer quelles perspectives s'offrent à nous, sur le plan de la collaboration internationale grâce à la mise sur cartes des éléments essentiels de notre travail.

Constatons, tout d'abord, que le matériel mis en ceurre est simple et assez pen coûteux surtout si on le compare au matériel électronique. Chaque chercheur pourrait donc, éventuellement, se procurer l'appareillage nécessaire mais je pense qu'on peut aussi envisager la création d'une centrale internationale chargée de regrouper toute la documentation et de la mettre sur cartes. Ces cartes pourraient être aisément consultées par correspondance puisqui on pourrait demander au centre les renseignements les plus divers et notamment des résultats d'analyses non publiés.

Dans d'autres domaines, on peut également envisager une collaboration internationale : par exemple pour la préparation des données servant à la confection de cartes permettant l'identification des pollens ou bien à la mise sur cartes de la répartition géographique des espèces intéressantes. Des copies des cartes pourraient ensuite être fournies à chacun.

\title{
CONCLUSIONS
}

Le tour d'horizon auquel nous venons de procéder n'a pas la prétention d'être complet. Bien des problèmes se posent à nous et dont il n'a pas été question ici. J'espère qu'une abondante discussion interviendra et que les lacunes de cet exposé se trouveront comblées par vos interventions. Je souhaite surtout que de ces réunions sortent de nourelles perspectives d'arenir pour l'analyse pollinique des miels.

\author{
RÉSUMÉ: \\ LTTILISATION DES CARTES I'ERFOREES \\ POLR L'ANAIYSE POLLINIQQLE DES MIELS
}

L'auteur expose les possibilités offertes par un système classique de cartes perforées (Sphinxo) pour le classement des données fournies par l'analyse pollinique des miels. Ces cartes permettent de retrouver très rapidement tous les niels présentant un spectre pollinique déterminé. Elles sont également utilisables pour plusieurs autres types de recherches.

\section{SUMMART}

THE USE OF PENCHED CARDS FOR THE POLLEN ANALYSIS OF HONEYS

The author lists the possibilities offered by a classical system of punched cards (Sphinxo) for the classification of data obtained from the pollen analysis of honeys. The use of these cards would make it possible to search out extremely quickly all the honevs having any given pollen properties. These cards could also be used for several other types of research. 